\title{
Simple preparation of thiol-ene particles in glycerol and surface functionalization by thiol-ene chemistry (TEC) and surface chain transfer free radical polymerization (SCT- FRP)
}

Hoffmann, Christian; Chiaula, Valeria; Yu, Liyun; Pinelo, Manuel; Woodley, John; Daugaard, Anders Egede

Published in:

Macromolecular Rapid Communications

Link to article, DOI:

10.1002/marc.201700394

Publication date:

2018

Document Version

Peer reviewed version

Link back to DTU Orbit

Citation (APA):

Hoffmann, C., Chiaula, V., Yu, L., Pinelo, M., Woodley, J., \& Daugaard, A. E. (2018). Simple preparation of thiolene particles in glycerol and surface functionalization by thiol-ene chemistry (TEC) and surface chain transfer free radical polymerization (SCT-FRP). Macromolecular Rapid Communications, 39(2), [1700394]. https://doi.org/10.1002/marc.201700394

\section{General rights}

Copyright and moral rights for the publications made accessible in the public portal are retained by the authors and/or other copyright owners and it is a condition of accessing publications that users recognise and abide by the legal requirements associated with these rights.

- Users may download and print one copy of any publication from the public portal for the purpose of private study or research.

- You may not further distribute the material or use it for any profit-making activity or commercial gain

- You may freely distribute the URL identifying the publication in the public portal 
DOI: 10.1002/marc.((insert number)) ((or ppap., mabi., macp., mame., mren., mats.))

\section{Communication}

Simple preparation of thiol-ene particles in glycerol and surface functionalization by thiol-ene chemistry (TEC) and surface chain transfer free radical polymerization (SCT-FRP)

Christian Hoffmann, Valeria Chiaula, Manuel Pinelo, John M. Woodley, Anders E. Daugaard*

Christian Hoffmann

Danish Polymer Centre, Department of Chemical and Biochemical Engineering, Technical

University of Denmark, Søltofts Plads Building 229, 2800 Kgs. Lyngby, Denmark

Valeria Chiaula

Danish Polymer Centre, Department of Chemical and Biochemical Engineering, Technical University of Denmark, Søltofts Plads Building 229, 2800 Kgs. Lyngby, Denmark

Associate Prof. Manuel Pinelo

Center for BioProcess Engineering, Department of Chemical and Biochemical Engineering, Technical University of Denmark, Søltofts Plads Building 229, 2800 Kgs. Lyngby, Denmark

Prof. John M. Woodley

Process and Systems Engineering Center (PROSYS), Department of Chemical and Biochemical Engineering, Technical University of Denmark, Søltofts Plads Building 229, 2800 Kgs. Lyngby, Denmark

Associate Prof. Anders E. Daugaard

Danish Polymer Centre, Department of Chemical and Biochemical Engineering, Technical University of Denmark, Søltofts Plads Building 229, 2800 Kgs. Lyngby, Denmark E-Mail: adt@kt.dtu.dk

\section{Abstract}

Thiol-ene (TE) based polymer particles have traditionally been prepared via emulsion polymerization in water (using surfactants, stabilizers and co-solvents). Here, we present a green and simple alternative with excellent control over particle size, while avoiding the addition of stabilizers. Glycerol is applied as a dispersing medium for the preparation of offstoichiometric TE (OSTE) microparticles, where sizes in the range of 40 to $400 \mu \mathrm{m}$ are obtained solely by changing the mixing speed of the emulsions prior to cross-linking. Control over surface chemistry is achieved by surface functionalization of excess thiol groups via 
photochemical thiol-ene chemistry (TEC) resulting in a functional monolayer. In addition, surface chain transfer free radical polymerization (SCT-FRP) was used for the first time to introduce a thicker polymer layer on the particle surface.

The application potential of the system is demonstrated by using functional particles as a support for immobilized enzymes in a continuous plug-flow reactor.

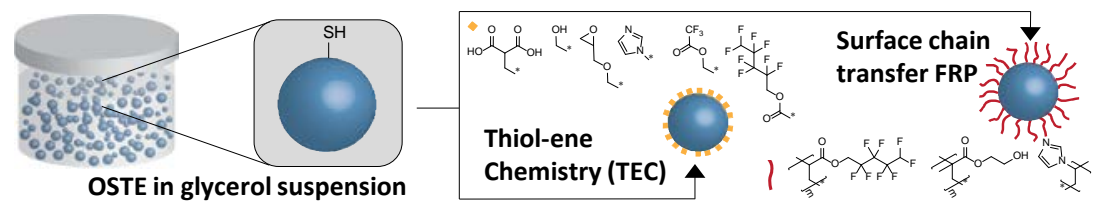




\section{Introduction}

During the past decades thiol-ene chemistry (TEC) has received increased attention for the preparation and modification of novel polymer systems. ${ }^{[1]}$ Radical mediated TEC or thiolMichael addition under basic conditions allows the reaction between a thiol group and a carboncarbon double bond (so called “ene”). ${ }^{[2]}$ Radical TEC can be initiated in various ways, such as photochemically, thermally or even electrochemically. High yields can be achieved within short reaction times under mild reaction conditions, which make TEC very robust and efficient. By applying a large variety and combination of different reactive monomers, various polymer architectures, such as linear, branched and cross-linked systems can be prepared. ${ }^{[3]}$ This versatility of network structures can be further increased by variations in monomer stoichiometry leading to excellent control over mechanical properties. ${ }^{[4]}$ The potential of this modularity has been demonstrated by applying stoichiometric thiol-ene (STE) and furthermore off-stoichiometric thiol-ene (OSTE) materials for the preparation of microfluidic systems ${ }^{[5-7]}$, hydrogels $^{[8,9]}$, microcapsules ${ }^{[10]}$ and high internal phase emulsions (HIPE). ${ }^{[1]}$ The stoichiometric imbalance between functional groups in the OSTE system results in unreacted thiol or ene groups within the polymer structure as well as on surfaces and can therefore directly be used for surface modification via TEC. ${ }^{[12-14]}$ Thus, hydrophilic and hydrophobic functional groups ${ }^{[15,16]}$ and biological moieties ${ }^{[17]}$ can easily be introduced, as well as more specific groups, such as maleic anhydride ${ }^{[18]}$ and cysteamine ${ }^{[19]}$, which have been applied for immobilization of enzymes.

The high versatility and modularity of TEC has also allowed the preparation of well-defined polymer particles with controlled properties, such as surface functionality, particle size, and thermal and mechanical behavior. Polymer particles are attractive due to their large application spectrum, such as drug-delivery, paints and coatings, chromatography and catalyst supports. ${ }^{[20,21]} \mathrm{TE}$ particles are typically prepared by suspension polymerization in which the monomers, facilitated by the use of different stabilizers and co-solvents, form initially an 
emulsion in water. Following polymerization, well-defined particles are obtained, as shown by Durham et al., who investigated the effect of different monomer combinations, stabilizers and stabilizer concentrations, ${ }^{[22-25]}$ as well as the effects of different modes of initiation, such as photochemical, thermal and redox initiation and their influence on particle size. ${ }^{[26]}$ Similarly, porous TE and thiol-alkyne particles were prepared using different porogens. ${ }^{[27,28]}$ Jasinski et al. used photoinitiated TEC for the preparation of nanoparticles from linear polymers. ${ }^{[29]}$ Furthermore, the investigation of different monomer combinations and the addition of further bulk functionalizations has been attempted in order to prepare functional TE particles. ${ }^{[30]}$ Likewise thiol-Michael addition has been used for the formation of monodisperse particles at the micrometer scale. ${ }^{[31,32]}$ It has also been demonstrated that the surface of OSTE particles, containing excess thiol or ene groups, can be functionalized with fluorescent moieties by thiolMichael reaction under basic conditions, photochemical TEC or thiol-isocyanate reaction. ${ }^{\text {[3-35] }}$ However, surfactants as well as organic co-solvents are generally necessary for the formation of polymer particles via suspension polymerization. The objective of this study was the development of a simple method for the controlled formation of OSTE particles without using surfactants or organic co-solvents by replacing water with glycerol as the dispersing medium. Post-preparation surface functionalization of the particles was demonstrated via photochemical TEC and by surface chain transfer free radical polymerization (SCT-FRP) in order to introduce well-defined functional groups on the surface. The obtained particles exhibited high control over size and surface chemistry, facilitating a broad range of application possibilities, which was demonstrated through the use of epoxide functional OSTE particles as support for the immobilization of enzymes in a biocatalytic plug-flow reactor.

\section{Results and Discussion}

\subsection{Preparation of OSTE particles in glycerol}

In a surfactant and organic co-solvent free process, off-stoichiometric thiol-ene (OSTE) particles were prepared by suspension polymerization in glycerol, which is a byproduct of 
biodiesel production and therefore a cheap, green and environmentally friendly alternative to water. ${ }^{[36]}$ OSTE particles (90\% thiol excess) were prepared by one-pot mixing of the monomers pentaerythritol tetrakis(3-mercaptopropionate) (PETMP) and 1,3,5-triallyl-1,3,5-triazine2,4,6(1H,3H,5H)-trione (TATATO) and the photoinitiator TPO-L in glycerol in a high speed mixer, as shown in Figure 1A.

Mixing speeds in the range of 1000 to $3500 \mathrm{rpm}$ were applied in order to obtain OSTE in glycerol emulsions, followed by photochemical cross-linking. The resulting microparticles were characterized by dynamic light scattering (DLS) and microscopy. As illustrated in Figure 1B, the applied mixing speed could be used to control the particle size. The particle size, depicted here as median of the volume distribution (Dv50) obtained from DLS, decreased with increasing mixing speeds from $402 \pm 43 \mu \mathrm{m}$ to $40.4 \pm 3.6 \mu \mathrm{m}$. Additionally, the size distributions (span) of the formed particles (between 1.1 and 2.4) was very similar to those obtained from traditional processes in water, in which sodium dodecyl sulfate (SDS) or sodium dodecyl benzene sulfate (SDBS) as surfactants and water as a dispersion phase were used. ${ }^{[23]}$ Scanning electron microscopy (SEM) images of the particles (see Figure 1B) confirmed the spherical shape of the prepared particles as well as the decreasing size of the particles as a function of mixing speed. The completely dried particles measured with SEM exhibit a reduced size. However, optical microscopy images (see Figure S1) of the particles dispersed in ethanol corroborated the measured size with DLS, which is attributed to substantial swelling in ethanol.

\subsection{Surface functionalization of OSTE particles via TEC and SCT-FRP}

The prepared particles were stable for up to three months in glycerol, and could directly be functionalized by photoinitiated TEC using different vinyl, allyl or (meth-)acrylic monomers as well as by SCT-FRP, as a novel pathway for surface modification of OSTE particles. These approaches demonstrated the capacity of excess surface thiols for versatile surface functionalization with control over different surface chemistries, as illustrated in Figure 2A. 
In the first approach, OSTE particles (prepared at a mixing speed of $1000 \mathrm{rpm}$ ) were surface functionalized via TEC introducing hydroxyl (AA), epoxide (AGE) and fluorinated groups (ATFA, OFPA). Additionally, pH responsive particle surfaces were achieved by grafting with Vim and AMA. Photochemical TEC was conducted at low monomer concentrations (0.3 M) in order to suppress potential polymerization reactions to occur. The IR spectrum of the virgin OSTE particles was characterized by alkane $\left(2960 \mathrm{~cm}^{-1}\right.$, C-H), thiol $\left(2569 \mathrm{~cm}^{-1}\right.$, S-H) carbonyl $\left(1730 \mathrm{~cm}^{-1}, \mathrm{C}=\mathrm{O}\right)$, alkene $(1678, \mathrm{C}=\mathrm{C})$ and ester $\left(1020 \mathrm{~cm}^{-1}, \mathrm{C}-\mathrm{O}-\mathrm{C}\right)$ stretches. TEC surface functionalized particles did not show any significant changes in their IR spectra, as shown in Figure S2. This was attributed to the penetration depth of the IR signal of several micrometers into th

e sample, which is known to result in very low intensity from thin surface layers. ${ }^{[37]}$ Consequently, the OSTE background dominated the spectrum. However, analysis via surface sensitive X-ray photoelectron spectroscopy (XPS) confirmed the surface modification with the individual reagents (see Table 1).

The atom composition of the virgin OSTE particles determined by XPS was in good agreement with the theoretical values. Surface TEC with ATFA and OFPA showed fluorine (F1s) contents of 17.3 atom\% and 9.0 atom\%, respectively, which confirmed the functionalization since fluorine was unique for these types of particles. Modification using Vim led to an increase in nitrogen content (4.4 N1s atom\%) compared to the original OSTE surfaces (3.0 N1s atom\%). The atom composition after surface modification with AGE, AA and AMA was identical to the reference OSTE particles, which is a result of similar theoretical atom compositions of these monomers and the OSTE material. Overall, short reaction times (5 min) and low monomer concentrations $(0.3 \mathrm{M})$ limited the thiol-ene reaction to a surface reaction forming a functional monolayer.

Conversely, by increasing the monomer concentration to classical polymerization conditions (1:2 $\mathrm{v} / \mathrm{v}$ monomers to ethanol) and extending the reaction time to $30 \mathrm{~min}$ it was possible to 
promote a more substantial grafting on the surface by SCT-FRP in order to achieve a thick and dense functional layer on the particle surface. In this way, it is possible to control the type of grafting reaction by tuning the reaction conditions, which was demonstrated by SCT-FRP with Vim, OFPA and HEMA. The monomers were polymerized in proximity to the particles under UV irradiation and IR analysis confirmed a significant grafting (see Figure S3). The spectrum of OSTE-g-pVim exhibited a sharp alkene stretch at $662 \mathrm{~cm}^{-1}$, which confirmed the introduced imidazole groups. The surface grafting with pOFPA and pHEMA was confirmed through the presence of C-F stretches $\left(806 \mathrm{~cm}^{-1}\right)$ typical for fluorinated compounds and hydroxyl stretches (3465 $\left.\mathrm{cm}^{-1}, \mathrm{O}-\mathrm{H}\right)$, respectively. Additionally, increased intensity of the C-O band at 1129 and $1143 \mathrm{~cm}^{-1}$, originating from the ester in the pendant group confirmed the surface grafting. Furthermore, XPS analysis showed substantial changes in atom composition for SCT-FRP functionalized particles, which were significantly higher than the corresponding particles prepared by TEC (see Table 1). The substantial decrease in sulfur content (S1s) in each case (pVim, pOFPA, and pHEMA) indicated the formation of a thicker surface layer. pHEMA grafted particles showed increased oxygen and carbon content, as well as a significant reduction in nitrogen content (2.2 atom\% N1s), confirming the pHEMA grafting. Surface grafting with pVim and pOFPA resulted in significant increases in nitrogen (9.0 atom\% N1s) and fluorine (44.4 atom\% F1s) content, respectively, compared to the reference OSTE surface. Thermogravimetric analysis (TGA) of the pristine and functionalized OSTE particles confirmed the thicker grafting resulting from the SCT-FRP compared to the TEC grafting. The thermograms of SCT-FRP functionalized particles all showed significant differences in thermal stability compared to the pristine and TEC modified OSTE particles, where especially pVim grafted particles had a weight loss of $15 \mathrm{wt} \%$ (see Figure S4). These results confirmed that the grafting density can be controlled by selecting TEC conditions for monolayer formation or grafting of a denser layer via SCT-FRP. 
Furthermore, the effect of functionalization onto the particle size was evaluated by SEM and DLS. SEM measurements confirmed that particle functionalization through either TEC or SCTFRP did not affect the particle size (see Figure S5 and S6). Additionally, a swelling study of the pristine and functionalized particles was conducted in ethanol and water in order to determine the swelling behavior as a function of the surface chemistry (see Figure 2 B). The pristine OSTE particles displayed a very similar median size (Dv50) in both ethanol (353 $\mu \mathrm{m})$ and water $(408 \mu \mathrm{m})$. Statistical ANOVA analysis using a significance level $\leq 0.05$ revealed a minor reduction in particle size in both, ethanol and water, upon TEC functionalization. Compared to the pristine OSTE, Vim, ATFA and OFPA functional particles could be considered similar when swollen in ethanol, whereas AMA, AGE and AA showed further decrease in size. This general tendency was statistically more pronounced in water, where all functionalized particles, besides OSTE-Vim, decreased significantly in size compared to the pristine one. This general size reduction in both ethanol and water was attributed to the newly introduced functional moieties that prevented the penetration of solvent into the particle core and thereby limited the swelling of the particles.

Compared to functionalizations by TEC, SCT-FRP with Vim, OFPA or HEMA resulted in particles with a significant reduction in size when measured in ethanol (120 to $174 \mu \mathrm{m})$. A reference experiment without initiator and UV irradiation did not show any changes in particle size, which confirmed that the reduction in size was a result of functionalization and not abrasion from the agitator. The size of the particles was highly dependent on the type polymer graft, where pHEMA grafted particles had similar sizes in water and ethanol. In contrast, OSTEpVim particles submerged in water exhibited a much higher degree of swelling (293 $\mu \mathrm{m})$ compared to the swelling in ethanol $(173 \mu \mathrm{m})$, which could be explained by protonation of imidazole moieties in water and the resultant repulsive interactions between the functional grafts leading to an increased thickness of the grafting layer. Likewise pOFPA grafted particles were observed to exhibit significantly different sizes in water and ethanol with a particle size 
of $447 \mu \mathrm{m}$ in water, almost 4-fold higher than the corresponding particle size in ethanol. The difference in size was attributed to a high degree of agglomeration as a result of the hydrophobic nature of the pOFPA, which was also observed visually.

\subsection{Functional OSTE particles applied as adsorbents for copper and as enzyme support in a plug-flow reactor}

The large amount of different functionalities, introduced via TEC or SCT-FRP, opens up for a broad range of applications. Imidazole groups for instance, are well-known to form a stable complex with metal ions and are therefore used as functionalities of different materials for the removal of heavy metals. ${ }^{[40,41]}$ In order to demonstrate their potential, imidazole functional particles, OSTE-Vim and OSTE-pVim, were used for the adsorption of $\mathrm{Cu}(\mathrm{II})$ ions. After incubation in a $\mathrm{CuSO}_{4}$ solution, the particles were dried and analyzed by SEM using an EDX detector (see Figure S7). The monolayer functional OSTE-Vim particles did not show a significant $\mathrm{Cu}(\mathrm{II})$ content, whereas OSTE-pVim particles contained around $2.7 \mathrm{w} \%$ of $\mathrm{Cu}(\mathrm{II})$ and thereby demonstrate a significant $\mathrm{Cu}(\mathrm{II})$ uptake, which was also clearly visible by the blue color of the SCT-FRP grafted particles compared to the colorless TEC particles. Furthermore, these results show the substantial effect of a thick surface layer formed through SCT-FRP compared to the TEC functionalization through an increase in metal adsorption.

Additionally, TEC functionalized particles were used as a support for enzyme immobilization in a biocatalytic plug-flow reactor. Epoxide groups are known to covalently bind enzymes through direct reaction with amine and thiol groups, which are found in the lysine and cysteine residues on the surface of the protein structure. ${ }^{[38]}$ In this study, epoxide functional particles (OSTE-AGE), which were prepared by TEC modification by AGE of OSTE particles prepared at $1000 \mathrm{rpm}$ mixing speed, were incubated with horseradish peroxidase (HRP) serving as a model enzyme, as illustrated in Figure 3A.

After filling the reactor with HRP immobilized particles and thorough rinsing with PBS buffer, the enzymatic activity was tested continuously using a solution containing 2,2' -azino-bis(3- 
ethylbenzothiazoline-6-sulfonic acid) diammonium salt (ABTS, $1 \mathrm{mM}$ ) and hydrogen peroxide $\left(\mathrm{H}_{2} \mathrm{O}_{2}, 10 \mathrm{mM}\right)$ in phosphate buffer (pH 5, $\left.0.1 \mathrm{M}\right)$ as a colorimetric assay. ABTS (absorbance maximum at $340 \mathrm{~nm}$ ) could be oxidized by HRP in the presence of $\mathrm{H}_{2} \mathrm{O}_{2}$ to ABTS ${ }^{\bullet+}$, which resulted in a shift in absorbance maximum to $412 \mathrm{~nm}\left(\varepsilon_{412}=3.6 \times 10^{4} \mathrm{M}^{-1} \mathrm{~cm}^{-1}\right){ }^{[39]}$ Using a constant flowrate of $0.2 \mathrm{~mL} \mathrm{~min}^{-1}$ allowed sampling at regular time intervals. In Figure 3B, photographs of the reactor at 1, 10 and 60 minutes operation time are shown, where the substrate solution was continuously added at the top and samples were taken at the bottom. The green color, which is formed after 1 min only at the top and over the entire reactor at 10 and 60 min, corresponds to the formed product. The absorbance of the withdrawn samples corresponding to the formed $\mathrm{ABTS}^{\bullet+}$ was determined and monitored over time, as shown in Figure 3C (full squares). Once the residence time within the plug-flow reactor of $6 \mathrm{~min}$ was reached, the absorbance from the product measured at the outlet increased significantly. After 15 minutes, steady state conditions were reached demonstrating stable enzymatic activity over the entire operation time. Reference particles (OSTE-AGE particles without HRP) were also tested, and are represented as empty squares in the Figure 3C, showing no enzymatic activity at any time. These results demonstrate the successful application of the prepared and epoxide functionalized OSTE particles in a biocatalytic application.

\section{Conclusions}

In this study, we have presented a novel, green and simple method for the preparation of OSTE particles in a controlled fashion via suspension polymerization in glycerol without using stabilizers, surfactants or co-solvents. Particle sizes were controlled by varying the mixing speed of the suspension prior to cross-linking via photoinitiated TEC. A variety of different surface chemistries were introduced in a post-preparation step via surface TEC or SCT-FRP. By TEC a functional monolayer was formed, which resulted in a minor decrease in particle size, when submerged in ethanol or water. However, thicker functional grafts formed as a result of 
the SCT-FRP approach which showed substantially reduced swelling in ethanol and increased swelling and agglomeration tendencies in water. The complementary nature of the two approaches enables simple control over surface grafting and surface chemistries depending on the specific application of the particles. Finally, TEC and SCT-FRP functionalized particles were used as adsorbents for copper, demonstrating the different impact of the two approaches. Additionally, TEC functionalized epoxide particles were successfully applied as support material for the immobilization of HRP, which was demonstrated in a biocatalytic plug-flow reactor. The ease of particle preparation and controlled surface functionalization opens up for a broad range of application possibilities.

\section{Supporting Information}

Supporting Information is available from the Wiley Online Library or from the author.

Acknowledgements: The authors wish to thank the Aage and Johanne Louis-Hansens Endowment for financial support.

Received: Month XX, XXXX; Revised: Month XX, XXXX; Published online:

((For PPP, use “Accepted: Month XX, XXXX" instead of "Published online”)); DOI: 10.1002/marc.((insert number)) ((or ppap., mabi., macp., mame., mren., mats.))

Keywords:

Thiol-ene chemistry, polymer particles, suspension polymerization, surface functionalization, enzyme immobilization

[1] A. B. Lowe, Polym. Chem. 2010, 1, 17.

[2] C. E. Hoyle, C. N. Bowman, Angew. Chemie 2010, 49, 1540. 
[3] A. B. Lowe, Polym. Chem. 2014, 5, 4820.

[4] C. F. Carlborg et al., J. Polym. Sci. Part A Polym. Chem. 2014, 52, 2604.

[5] C. F. Carlborg, T. Haraldsson, K. Öberg, M. Malkoch, W. van der Wijngaart, Lab Chip 2011, 11, 3136.

[6] P. Mazurek, A. E. Daugaard, M. Skolimowski, S. Hvilsted, A. L. Skov, RSC Adv. 2015, 5, 15379.

[7] S. M. Tähkä et al., J. Chromatogr. A 2015, 1426, 233.

[8] A. A. Aimetti, A. J. Machen, K. S. Anseth, Biomaterials 2009, 30, 6048.

[9] T. Yang et al., J. Polym. Sci. Part A Polym. Chem. 2011, 49, 4044.

[10] D. V. Amato, H. Lee, J. G. Werner, D. A. Weitz, D. L. Patton, ACS Appl. Mater. Interfaces 2017, 9, 3288.

[11] E. Lovelady, S. D. Kimmins, J. Wu, N. R. Cameron, Polym. Chem. 2011, 2, 559.

[12] D. Wasserberg et al., J. Mater. Chem. 2012, 22, 16606.

[13] J. Zhang, Y. Chen, M. A. Brook, Langmuir 2013, 29, 12432.

[14] K. Y. Tan, M. Ramstedt, B. Colak, W. T. S. Huck, J. E. Gautrot, Polym. Chem. 2016, 7, 979.

[15] G. Pardon et al., Microfluid. Nanofluidics 2014, 17, 773.

[16] C. F. Carlborg, F. Moraga, F. Saharil, W. van der Wijngaart, T. Haraldsson, Proc. Micro Total Anal. Syst. 2012, 677, at <http://www.rsc.org/images/loc/2012/pdf/M.5.137.pdf>.

[17] N. A. Feidenhans'l, J. P. Lafleur, T. G. Jensen, J. P. Kutter, Electrophoresis 2014, 35, 282.

[18] E. Çakmakçi, B. Yuce-Dursun, S. Demir, React. Funct. Polym. 2017, 111, 38.

[19] J. P. Lafleur et al., Lab Chip 2015, 15, 1.

[20] H. Kawaguchi, Prog. Polym. Sci. 2000, 25, 1171.

[21] J. A. Champion, Y. K. Katare, S. Mitragotri, J. Control. Release 2007, 121, 3. 
[22] O. Z. Durham, S. Krishnan, D. A. Shipp, ACS Macro Lett. 2012, 1, 1134.

[23] O. Z. Durham, D. A. Shipp, Polymer 2014, 55, 1674.

[24] O. Z. Durham, D. A. Shipp, Colloid Polym. Sci. 2015, 293, 2385.

[25] O. Z. Durham, D. V. Chapman, S. Krishnan, D. A. Shipp, Macromolecules 2017, 50, 775.

[26] F. Alimohammadi et al., Polymer 2016, 105, 180.

[27] J. Tan et al., Rsc Adv. 2014, 4, 13334.

[28] S. Cai et al., Polym. Chem. 2016, 7, 7400.

[29] F. Jasinski et al., ACS Macro Lett. 2014, 3, 958.

[30] C. Wang et al., Adv. Funct. Mater. 2017, 27.

[31] C. Wang, M. Podgórski, C. N. Bowman, Mater. Horizons 2014, 1173, doi:10.1073/pnas.1201800109.

[32] C. Wang et al., Macromolecules 2015, 48, 8461.

[33] D. V. Amato, D. N. Amato, A. S. Flynt, D. L. Patton, Polym. Chem. 2014, 6, 5625.

[34] D. N. Amato et al., Chem. Commun. 2015, 51, 10910.

[35] O. Z. Durham, H. R. Norton, D. A. Shipp, RSC Adv. 2015, 5, 66757.

[36] C. A. G. Quispe, C. J. R. Coronado, J. A. Carvalho Jr, Renew. Sustain. Energy Rev. 2013, 27, 475 .

[37] C. Y. Tang, Y. N. Kwon, J. O. Leckie, J. Memb. Sci. 2007, 287, 146.

[38] E. Cakmakci, O. Danis, S. Demir, Y. Mulazim, M. V. Kahraman, J. Microbiol. Biotechnol. 2013, 23, 205.

[39] B. R. E. Childs, W. G. Bardsley, Biochem. J. 1975, 145, 93.

[40] T. Kang, Y. Park, K. Choi, J. S. Lee, J. Yi, J. Mater. Chem. 2004, 14, 1043.

[41] K. Tekin, L. Uzun, Ç. A. Şahin, S. Bektaş, A. Denizli, React. Funct. Polym. 2011, 71, 985. 

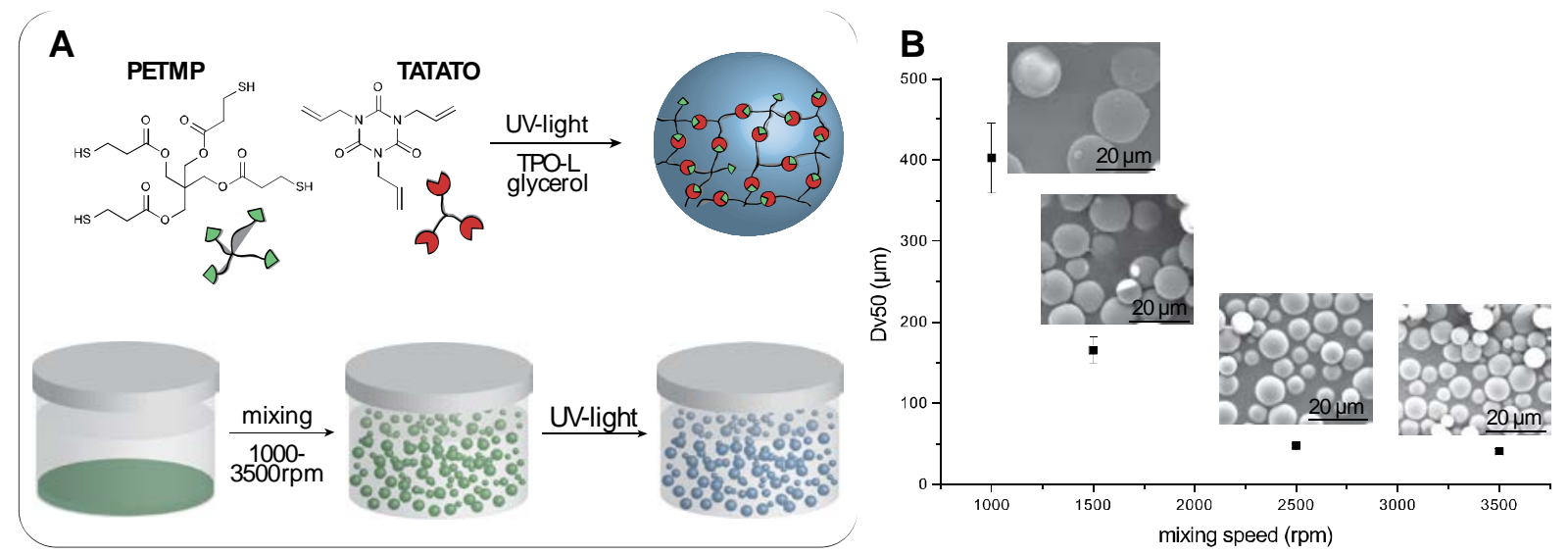

Figure 1. A) Preparation of OSTE microparticles via suspension in a speedmixer followed by subsequent photochemical cross-linking of PETMP and TATATO. B) Particle size (Dv50) as a function of mixing speed with optical microscopy images (50 x magnification) of the OSTE particles. 


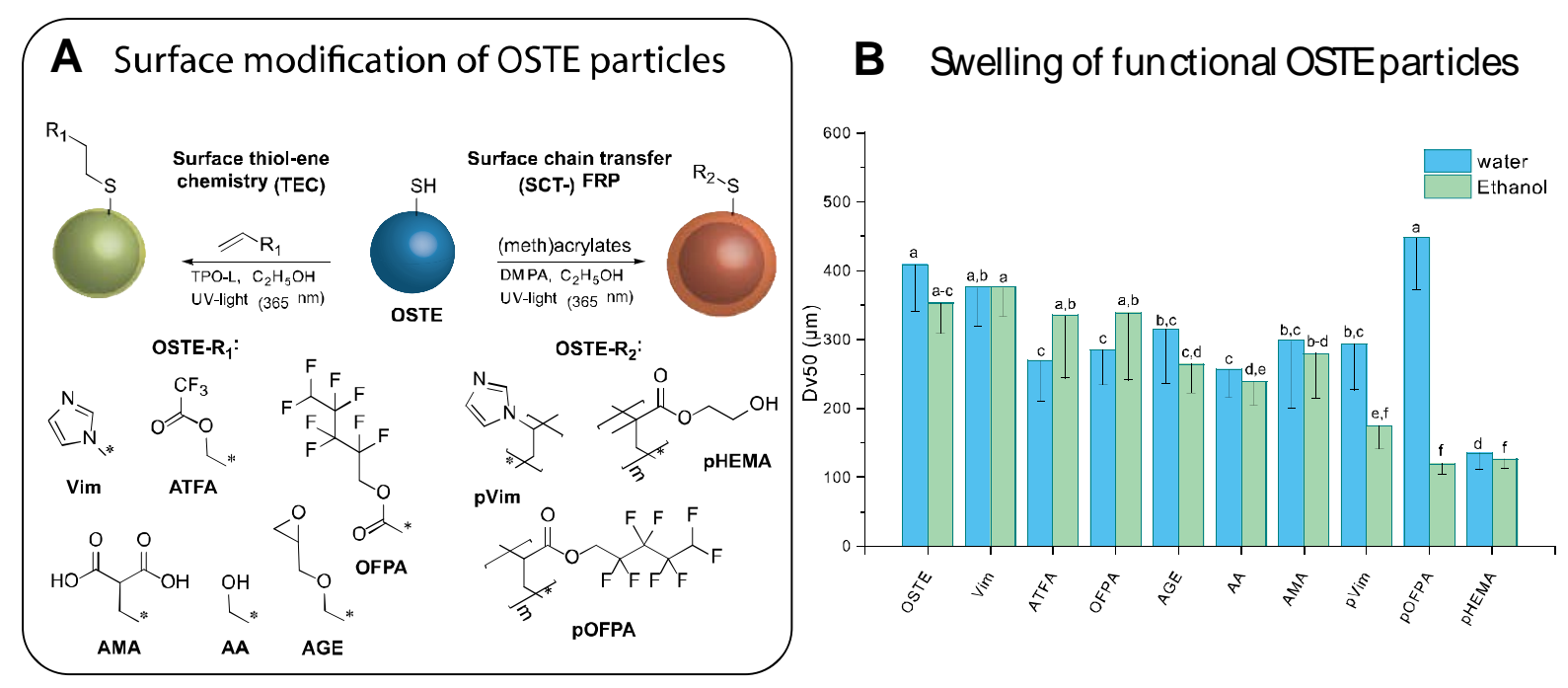

Figure 2. A) Surface functionalization of OSTE particles via two different routes, left: surface TEC with different reagents, such as 1-vinyl imidazole (Vim), allyl trifluoroacetate (ATFA), 2,2,3,3,4,4,5,5 octylfluoropentylacrylate (OFPA), allyl malonic acid (AMA), allyl alcohol (AA), and allyl glycidyl ether (AGE) at low concentration (0.3 M) leading to a functional monolayer on the surface; right: SCT-FRP with various monomers, such as Vim, 2hydroxyethyl methacrylate (HEMA) and OFPA at high concentration (0.7 - 2.4 M), leading to a functional polymer grafted surface. B) Swelling study of OSTE particles containing various surface functionalities grouped by ANOVA analysis (letters above bars, $\mathrm{p}=0.05$ ) in ethanol and water individually. 
Table 1. XPS data of pristine and surface functionalized OSTE particles via TEC ${ }^{\mathrm{a}}$ and SCTFRP $^{\mathrm{b}}$

\begin{tabular}{llllll}
\hline particle type & $\begin{array}{l}\text { C1s } \\
\text { [atom\%] }\end{array}$ & $\begin{array}{l}\text { O1s } \\
\text { [atom\%] }\end{array}$ & $\begin{array}{l}\text { N1s } \\
\text { [atom\%] }\end{array}$ & $\begin{array}{l}\text { S2p } \\
\text { [atom\%] }\end{array}$ & $\begin{array}{l}\text { F1s } \\
\text { [atom\%] }\end{array}$ \\
\hline OSTE & $60.1 \pm 1.4$ & $26.6 \pm 0.9$ & $3.0 \pm 0.6$ & $10.3 \pm 0.3$ & - \\
OSTE-Vim $^{a}$ & $60.8 \pm 0.5$ & $24.2 \pm 0.8$ & $4.4 \pm 0.3$ & $10.6 \pm 0.8$ & - \\
OSTE-ATFA $^{a}$ & $52.8 \pm 0.4$ & $21.5 \pm 0.5$ & $2.4 \pm 0.4$ & $6.0 \pm 0.4$ & $17.3 \pm 0.7$ \\
OSTE-OFPA $^{a}$ & $55.5 \pm 1.0$ & $22.4 \pm 0.7$ & $4.1 \pm 0.5$ & $9.0 \pm 0.5$ & $9.0 \pm 1.7$ \\
OSTE-AGEa $^{a}$ & $62.3 \pm 0.3$ & $24.1 \pm 0.5$ & $3.5 \pm 0.5$ & $10.1 \pm 0.3$ & - \\
OSTE-AA $^{a}$ & $62.2 \pm 0.9$ & $23.6 \pm 0.7$ & $3.5 \pm 0.4$ & $10.7 \pm 0.2$ & - \\
OSTE-AMA $^{a}$ & $61.7 \pm 0.3$ & $24.4 \pm 0.5$ & $3.7 \pm 0.0$ & $10.2 \pm 0.5$ & - \\
OSTE-pVim $^{\mathrm{b}}$ & $63.8 \pm 0.9$ & $19.0 \pm 0.3$ & $9.0 \pm 0.3$ & $8.2 \pm 0.8$ & - \\
OSTE-pOFPA $^{\mathrm{b}}$ & $42.6 \pm 0.2$ & $11.1 \pm 0.5$ & $0.6 \pm 0.6$ & $1.4 \pm 0.2$ & $44.4 \pm 1.0$ \\
OSTE-pHEMA $^{\mathrm{b}}$ & $62.9 \pm 0.7$ & $28.3 \pm 0.5$ & $2.2 \pm 0.1$ & $6.6 \pm 0.1$ & -
\end{tabular}



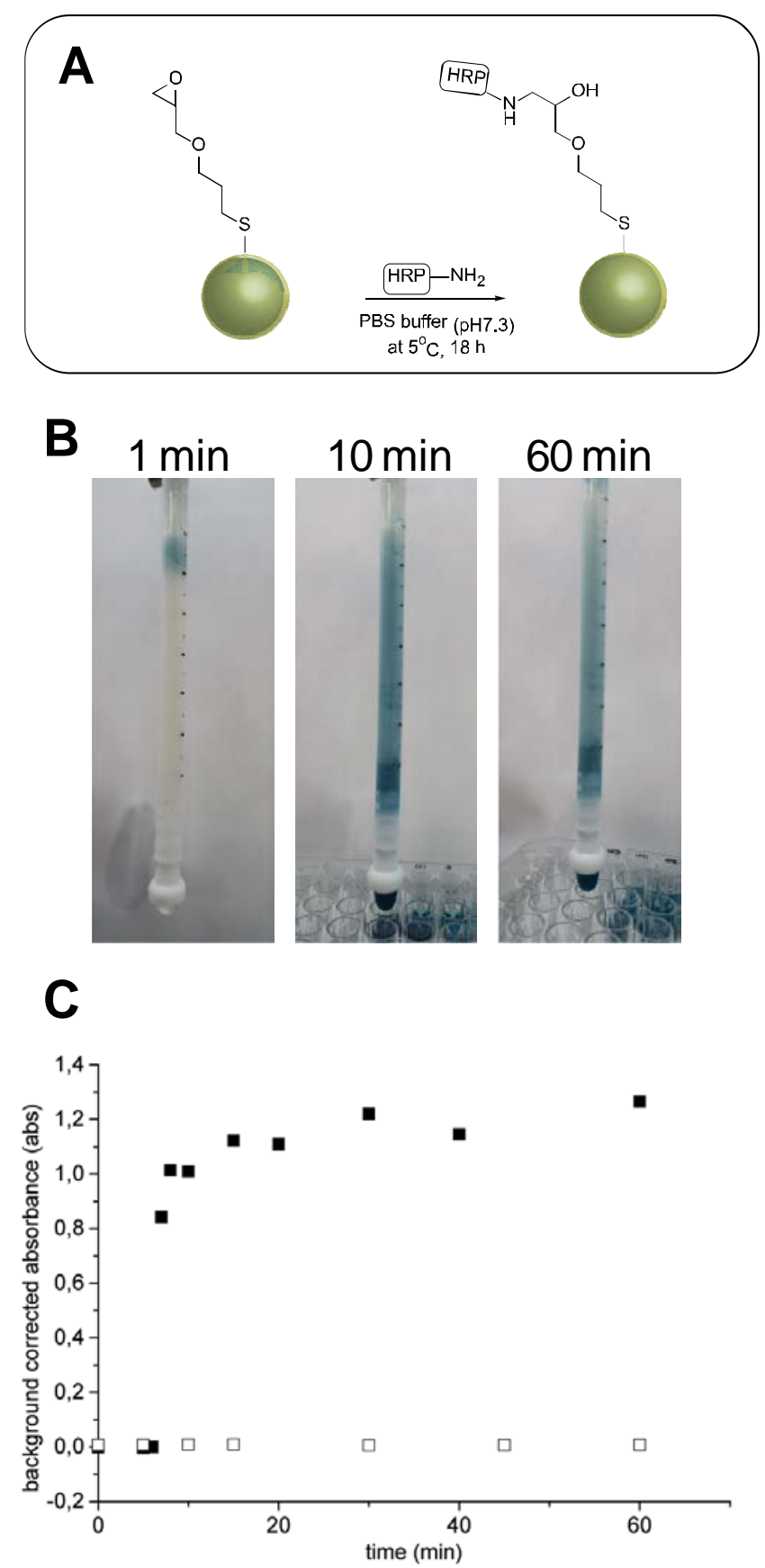

Figure 3. A) Immobilization of HRP on OSTE-AGE particles. B) Photographs of plug-flow reactor at $1 \mathrm{~min}, 10 \mathrm{~min}$ and $60 \mathrm{~min}$ of reaction time using a flow of $0.2 \mathrm{ml} \mathrm{min}^{-1}$ during a ABTS $/ \mathrm{H}_{2} \mathrm{O}_{2}$ activity measurement of immobilized HRP on OSTE-AGE particles. C) Enzyme activity as background corrected absorbance at $412 \mathrm{~nm}$ over time of OSTE-AGE particles with immobilized enzyme (full squares) and a reference (without enzyme -empty squares) 


\section{Table of contents entry}

A simple and fast process for the preparation of off-stoichiometric thiol-ene (OSTE) particles in glycerol without stabilizers or co-solvents is demonstrated. Particle sizes are controlled solely by changing mixing speeds. Versatile surface modification via thiol-ene chemistry (TEC) or surface chain transfer free radical polymerization (SCT-FRP) demonstrates the introduction of various functional groups and selectively changed the extent of grafting thickness.

Christian Hoffmann, Valeria Chiaula, Manuel Pinelo, John M. Woodley, Anders E. Daugaard*

Simple preparation of thiol-ene particles from glycerol and surface functionalization by thiol-ene chemistry (TEC) and surface chain transfer free radical polymerization (SCTFRP)

ToC figure

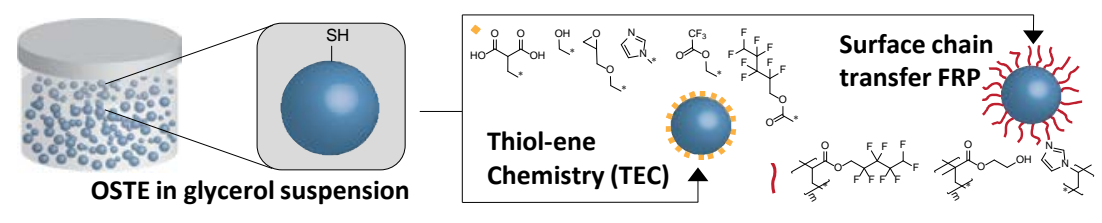




\section{Supporting Information}

\section{Simple preparation of thiol-ene particles from glycerol and surface functionalization by thiol-ene chemistry (TEC) and surface chain transfer free radical polymerization (SCT-FRP)}

Christian Hoffmann, Valeria Chiaula, Manuel Pinelo, John M. Woodley, Anders E. Daugaard*

\section{Experimental Section}

\subsection{Materials}

Pentaerythritol tetrakis(3-mercaptopropionate) (PETMP, >95\%), 1,3,5-Triallyl-1,3,5-triazine2,4,6(1H,3H,5H)-trione (TATATO, 98\%), allyl pentafluorobenzene (APFB, >99\%), allyltrifluoroacetate (ATFA , 98\%), allyl alcohol (AA, 99\%), allyl malonic acid (AMA, $\geq 98 \%$ ), 1-vinyl imidazole (Vim, $\geq 99 \%$ ), allyl glycidyl ether (AGE, >99\%), 2,2-dimethoxy-2phenylacetophenone (DMPA, 99\%), horseradish peroxidase (HRP, lyophilized powder, 50-150 U mg-1), 2,2'-Azino-bis(3-ethylbenzothiazoline-6-sulfonic acid) diammonium salt (ABTS, $\geq 98 \%)$ and hydrogen peroxide $\left(\mathrm{H}_{2} \mathrm{O}_{2}, 3 \%\right)$ were obtained from Sigma-Aldrich. 2-hydroxyethyl methacrylate (HEMA, 99\%) and 2,2,3,3,4,4,5,5 octafluoropentyl acrylate (OFPA, 97\%) were purchased from Sigma Aldrich and passed through a short plug flow column containing aluminum oxide (Sigma-Aldrich, activated, basic, Brockmann I, standard grade) prior to use. Lucirin TPO-L (ethyl-2, 4, 6-tri- methylbenzoylphenyl phosphinate) was obtained from BASF. Ethanol absolute (99.97\%) was purchased from VWR. Glycerol was kindly provided by Emmelev, Otterup A/S Denmark and was used as received.

\subsection{Methods}

Fourier transform infrared (FT-IR) spectroscopy was conducted using a Nicolet iS50 FT-IR fitted with a diamond crystal attenuated total reflection accessory (ATR), which operated at a resolution of $4 \mathrm{~cm}^{-1}$ and 32 scans per measurement. X-ray photoelectron spectroscopy (XPS) analysis was carried out on a Thermo Fisher Scientific K-Alpha (East Grinstead, UK). Large 
area surface analysis used a $400 \mu \mathrm{m}$ spot of monochromatized aluminum Ka radiation. Survey (pass energy $200 \mathrm{eV}$ ) and high-resolution (pass energy $50 \mathrm{eV}$ ) spectra for relevant elements were acquired of the particles. Data analyses of the obtained XPS spectra were performed using the Avantage software package as provided by the manufacturer. The shape and size of the particles was assessed by optical microscopy using a Leica DM LB optical microscope. Particle size distributions were determined by Dynamic Light Scattering (DLS) measurements, performed on a Malvern Mastersizer 3000 using laser diffraction method. The particles, dispersed in a fluid, passing through a focused red (or blue) laser beam in a quartz measurement cell scattered light at various angles. Generally, the median for volume distribution (Dv50), which divides the whole population in two equal parts, and the span were determined from 5 measurements. The span is calculated according to the following equation:

$$
\operatorname{Span}=\frac{\mathrm{D}_{\mathrm{v} 0.9}-\mathrm{D}_{\mathrm{v} 0.1}}{\mathrm{D}_{\mathrm{v} 0.5}}
$$

Enzyme activity measurements were conducted in a PS 96 well microplate using a POLARstar ${ }^{\circledR}$ Omega from BMG Labtech equipped with an UV-VIS probe (20 scans per measurement) at $25^{\circ} \mathrm{C}$.

Scanning electron microscopy (SEM) images were recorded using a FEI Quanta 200 ESEM FEG. Prior to testing, the particles were coated under vacuum and a current of $20 \mathrm{~mA}$ for $5 \mathrm{~s}$ resulting in a 3.9 nm-thick gold layer using a high resolution sputter coater Cressington 208HR. For the determination of the atom composition after $\mathrm{Cu}(\mathrm{II})$ adsorption onto OSTE-Vim and OSTE-pVim, particles were dried, coated with gold as previously described and analyzed with a FEI Inspect S SEM microscope using an energy-dispersive X-ray spectroscopy (EDX) detector (Oxford Instruments). Thermal analysis of the particles was conducted by thermogravimetric analysis (TGA) using a Discovery TGA from TA Instruments within a temperature range from 25 to $900{ }^{\circ} \mathrm{C}$ with a heating rate of $10 \mathrm{~K} \mathrm{~min}^{-1}$ under nitrogen flow.

\subsection{Preparation of off-stoichiometric thiol-ene (OSTE) particles in glycerol}


PETMP (0.736 g, 0.0015 mol), TATATO (0.264 g, 0.0011 mol), TPO-L (1.0 mg, $0.1 \mathrm{wt} \%$ ) and glycerol (10 mL) were added into a mixing cup under exclusion of light. Then, the mixture were homogenized by mixing in a Speedmixer at 1000 rpm (SpeedMixerTM DAC 150.1 FVZ) for 1.5 minutes. Subsequently, the mixture was exposed to UV light $\left(\lambda=365 \mathrm{~nm}, 1.4 \mathrm{~mW} \mathrm{~cm}^{-2}\right)$ for 10 minutes. Then, ethanol was added to the suspension, which was manually stirred and the resulting particles were filtered and thoroughly rinsed with ethanol. An aliquot of the particles was dried in vacuo for further characterization, whereas the main part was resuspended in ethanol for storage and further functionalization.

IR (cm-1): 2960 (C-H), 2569 (S-H), 1730 (C=O), 1678 (C=Calkene), 1459 (O-CH2 ester), 1140 (C-

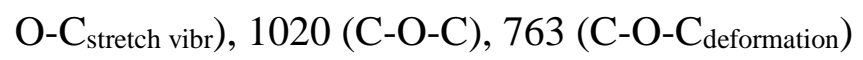

\subsection{General surface functionalization of OSTE particles via TEC using allyl malonic acid (OSTE-AMA)}

In a general procedure, $1.0 \mathrm{~g}$ of OSTE microparticles (obtained at $1000 \mathrm{rpm}$ ) was suspended in $10 \mathrm{ml}$ of ethanol. Under exclusion of light, allyl malonic acid (0.432 g, $3.0 \mathrm{mmol})$ and TPO-L (8.4 $\mu \mathrm{L}, 0.003 \mathrm{mmol}$ ) were added. Then, the suspension was stirred by magnetic stirring (300 $\mathrm{rpm})$ for $5 \mathrm{~min}$ and irradiated with $\mathrm{UV}$ light $\left(\lambda=365 \mathrm{~nm}, 0.4 \mathrm{~mW} \mathrm{~cm}{ }^{-2}\right)$. Subsequently, the particles were filtered and washed thoroughly with ethanol before resuspending them in ethanol (10 mL) for further use.

IR (cm $\left.{ }^{-1}\right): 2961$ (C-H), 2568 (S-H), 1730 (C=O), 1678 (C=Calkene), 1460 (O-CH2 ester), 1141 (C-

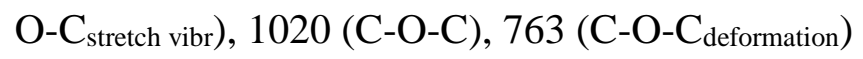

\subsubsection{Preparation of OSTE-AA}

OSTE-AA was prepared in accordance to the general procedure, using $10 \mathrm{~mL}$ of an ethanolic solution of allyl alcohol (204 $\mu \mathrm{L}, 3.0 \mathrm{mmol})$ and TPO-L $(8.4 \mu \mathrm{L}, 0.003 \mathrm{mmol})$ as reagent.

IR (cm $\left.{ }^{-1}\right): 2960$ (C-H), 2566 (S-H), 1730 (C=O), 1678 (C=Calkene), 1460 (O-CH2 ester), 1141 (C-

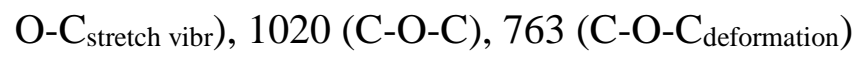

\subsubsection{Preparation of OSTE-Vim}


OSTE-Vim was prepared in accordance to the general procedure, using $10 \mathrm{~mL}$ of an ethanolic solution of 1-vinyl imidazole (Vim, $271 \mu \mathrm{L}, 3.0 \mathrm{mmol})$ and TPO-L (8.4 $\mu \mathrm{L}, 0.003 \mathrm{mmol})$ as reagent.

IR (cm $\left.{ }^{-1}\right): 2960$ (C-H), 2567 (S-H), 1730 (C=O), 1678 (C=Calkene), 1460 (O-CH2 ester), 1141 (C-

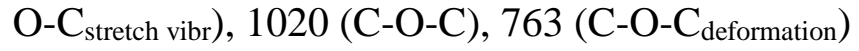

\subsubsection{Preparation of OSTE-ATFA}

OSTE-ATFA was prepared in accordance to the general procedure, using $10 \mathrm{~mL}$ of an ethanolic solution of allyl trifluoroacetate (ATFA, $391 \mu \mathrm{L}, 3.0 \mathrm{mmol}$ ) and TPO-L (8.4 $\mu \mathrm{L}, 0.003 \mathrm{mmol})$ as reagent.

IR (cm $\left.{ }^{-1}\right): 2960$ (C-H), 2566 (S-H), 1730 (C=O), 1678 (C=Calkene), 1460 (O-CH2 ester), 1140 (C-

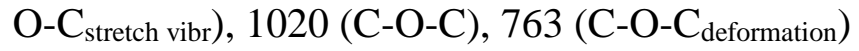

\subsubsection{Preparation of OSTE-OFPA}

OSTE-OFPA was prepared in accordance to the general procedure, using $10 \mathrm{~mL}$ of an ethanolic solution of 2,2,3,3,4,4,5,5 octafluoropentyl acrylate (OFPA, $583 \mu \mathrm{L}, 3.0 \mathrm{mmol}$ ) and TPO-L (8.4 $\mu \mathrm{L}, 0.003 \mathrm{mmol})$ as reagent.

IR (cm²): 2960 (C-H), 2568 (S-H), 1730 (C=O), 1678 (C=Calkene), 1460 (O-CH2 ester), 1141 (C-

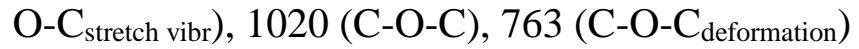

\subsubsection{Preparation of OSTE-AGE}

OSTE-AGE was prepared in accordance to the general procedure, using $10 \mathrm{~mL}$ of an ethanolic solution of allyl glycidylether (AGE, $360 \mu \mathrm{L}, 3.0 \mathrm{mmol}$ ) and TPO-L (8.4 $\mu \mathrm{L}, 0.003 \mathrm{mmol}$ ) as reagent.

IR (cm $\left.{ }^{-1}\right): 2960$ (C-H), 2567 (S-H), 1730 (C=O), 1678 (C=Calkene), 1460 (O-CH2 ester), 1140 (C-

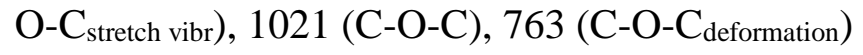

\subsection{General surface functionalization of OSTE particles via surface chain transfer free radical polymerization (SCT FRP) with 1-vinyl imidazole (OSTE-g-pVim)}


In a general procedure, $1 \mathrm{~g}$ of previously prepared OSTE particles were submerged in a solution containing 1-vinyl imidazole (Vim, $3.3 \mathrm{~mL}, 27.5 \mathrm{mmol}$ ), 2,2-dimethoxy-2-phenylacetophenone (DMPA, $47.2 \mathrm{mg}, 0.50 \mathrm{~mol} \%$ ) and ethanol (6.7 mL), which was screened from ambient light. Under magnetic stirring, this mixture was subsequently irradiated with UV light $(\lambda=365 \mathrm{~nm}$, $0.4 \mathrm{~mW} \mathrm{~cm}^{-2}$ ) for $30 \mathrm{~min}$. Then, the particles were filtered and thoroughly rinsed with ethanol before storing them in $10 \mathrm{~mL}$ of ethanol until further use.

IR (cm $\left.{ }^{-1}\right): 2955$ (C-H), 1729 (C=O), 1676 (C=Calkene), 1461 (O-CH2 ester), 1350, 1229 (N-H),

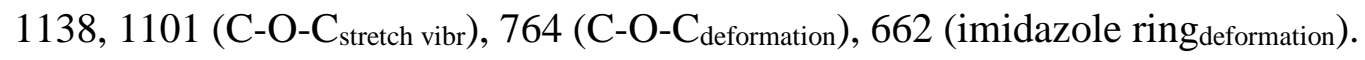

\subsubsection{Preparation of OSTE-g-pOFPA}

OSTE-g-pOFPA was prepared in accordance with the general procedure, using a solution containing OFPA (3.3 mL, $17.3 \mathrm{mmol}$ ), DMPA (22.2 mg, $0.5 \mathrm{~mol} \%$ ) and ethanol (6.7 mL). IR (cm $\left.{ }^{-1}\right)$ : 2961 (C-H), 1733 (C=O), 1681 (C=Calkene), 1461 (O-CH2 ester), 1129 (C-O stretch vibr), 989 (C-O), 902, 806 (C-F), 764 (C-O-C deformation$)$

\subsubsection{Preparation of OSTE-g-pHEMA}

OSTE-g-pHEMA was prepared in accordance with the general procedure, using a solution containing 2-hydroxylethyl methacrylate (HEMA, 3.3 mL, 27.5 mmol), DMPA (35.2 mg, 0.5 mol\%) and ethanol (6.7 mL).

IR (cm-1): 3465 (O-H), 2948 (C-H), 1728 (C=O), 1678 (C=Calkene), 1460 (O-CH2 ester), 1143 (C$\mathrm{O}_{\text {stretch vibr }),}$ 1021, 764 (C-O-C $\left.\mathrm{C}_{\text {deformation }}\right)$

\subsection{Adsorption of $\mathrm{Cu}(\mathrm{II})$ ions onto OSTE-Vim and OSTE-pVim particles}

A batch of previously prepared OSTE-Vim (48.8 mg) and OSTE-pVim particles (51.0 mg) was each submerged into $10 \mathrm{~mL}$ of a $\mathrm{CuSO}_{4}$ solution (1.26 mg mL ${ }^{-1}$, $\left.\mathrm{pH} 4.9\right)$. After an incubation time of 2 h under shaking, the solution was removed by filtration and the particles were thoroughly washed with deionized water followed by ethanol. The particles were stored in ethanol until further use. 


\subsection{Preparation and testing of a plug flow reactor with enzymatically active OSTE-AGE particles}

A batch of previously prepared OSTE-AGE particles was dried in vacuo. $0.8 \mathrm{~g}$ of these particles was redispersed in ethanol, which was solvent exchanged with PBS buffer (pH 7.3). The final amount of PBS buffer was $3.2 \mathrm{~mL}$ and $3.5 \mathrm{mg}$ of horseradish peroxidase (HRP) was subsequently added. This mixture was incubated under shaking at $22^{\circ} \mathrm{C}$ for $20 \mathrm{~h}$. Then, $1 \mathrm{~mL}$ of the supernatant was removed for further analysis and the particles were added into a tubular reactor with an inner diameter of $5.0 \mathrm{~mm}$. The filled reactor was rinsed with PBS buffer; first manually with $10 \mathrm{~mL}$ and then with a flow rate of $0.2 \mathrm{~mL} \mathrm{~min}^{-1}$ for $30 \mathrm{~min}$ using a NE-1000 single syringe pump. While washing with PBS buffer, a phosphate buffer solution (0.1M, pH5) containing ABTS $(1.0 \mathrm{mM})$ and hydrogen peroxide $\left(\mathrm{H}_{2} \mathrm{O}_{2}, 10 \mathrm{mM}\right)$ was added to the eluent at the outlet and after 20 min of flushing with $0.2 \mathrm{~mL} \mathrm{~min}^{-1}$ no enzyme activity in the eluent was observed. After flushing with PBS buffer a phosphate buffer solution (0.1 M, pH5) containing ABTS $(1.0 \mathrm{mM})$ and hydrogen peroxide $\left(\mathrm{H}_{2} \mathrm{O}_{2}, 10 \mathrm{mM}\right)$ was passed through the reactor with a flow rate of $0.2 \mathrm{~mL} \mathrm{~min}^{-1}$. At distinct time intervals, aliquots of the solution at the outlet were collected. 50 as well $100 \mu \mathrm{L}$ of these samples were transferred into a polystyrene (PS) 96 microplate and the absorbance was measured at $412 \mathrm{~nm}$ corresponding to the absorbance maximum of the oxidized product $\left(\mathrm{ABTS}^{\bullet+}\right)$.

\section{Supporting Figures}



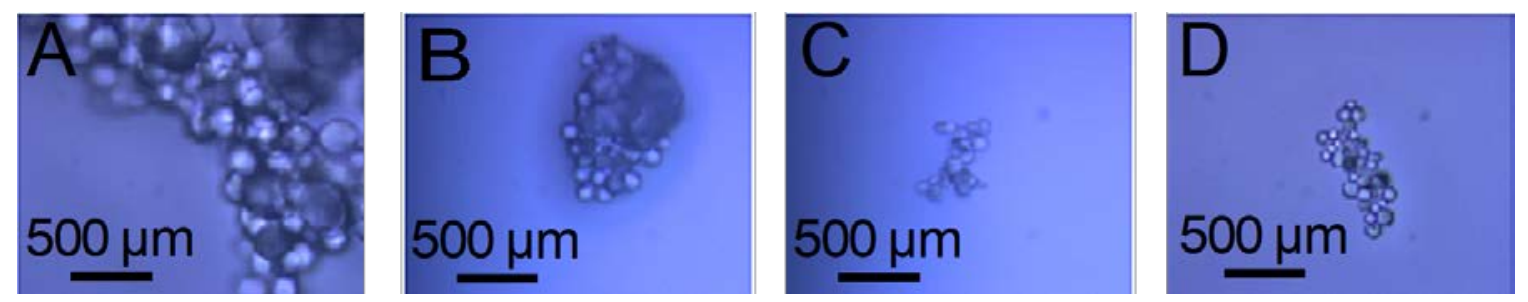

Figure S1 Optical microscopy images of OSTE particles prepared at different mixing speeds,

A) $1000 \mathrm{rpm}$, B) $1500 \mathrm{rpm}$, C) $2500 \mathrm{rpm}$, and D) $3500 \mathrm{rpm}$

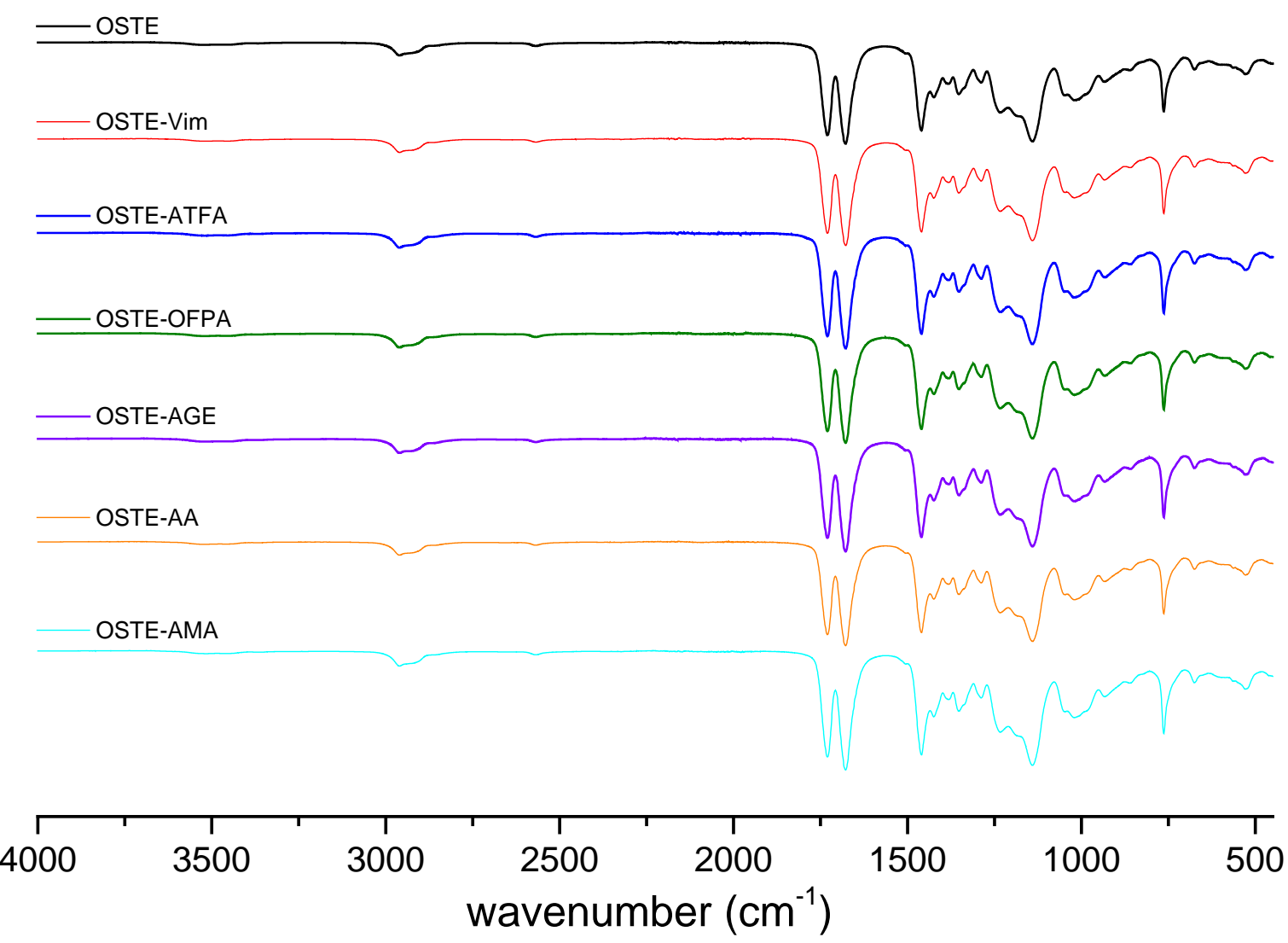

Figure S2 IR spectra of surface functionalized OSTE particles via TEC using various allylic and vinylic reagents 


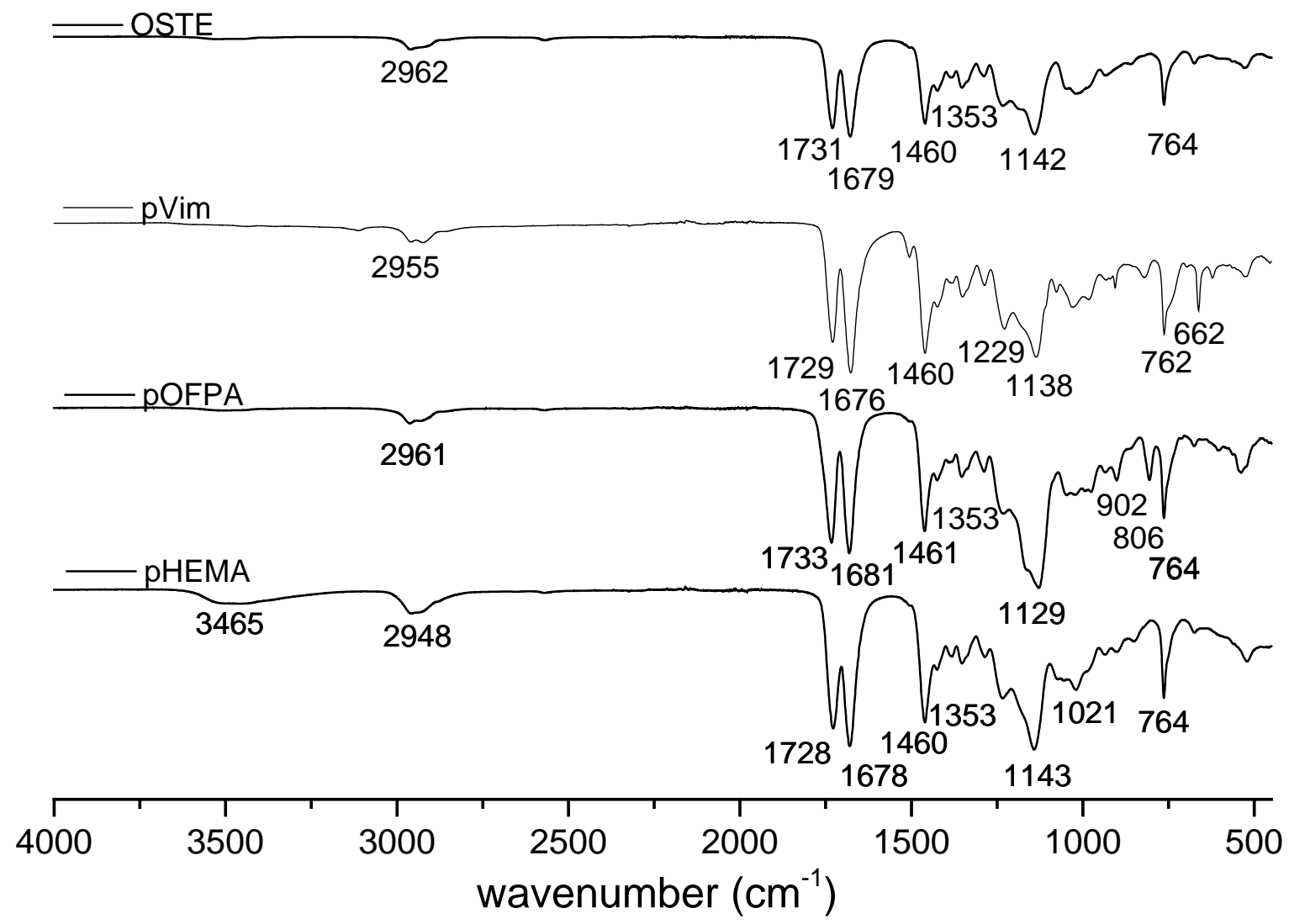

Figure S3 IR spectra of surface functionalized OSTE particles via SCT-FRP using vinylic, acrylic and methacrylic reagents
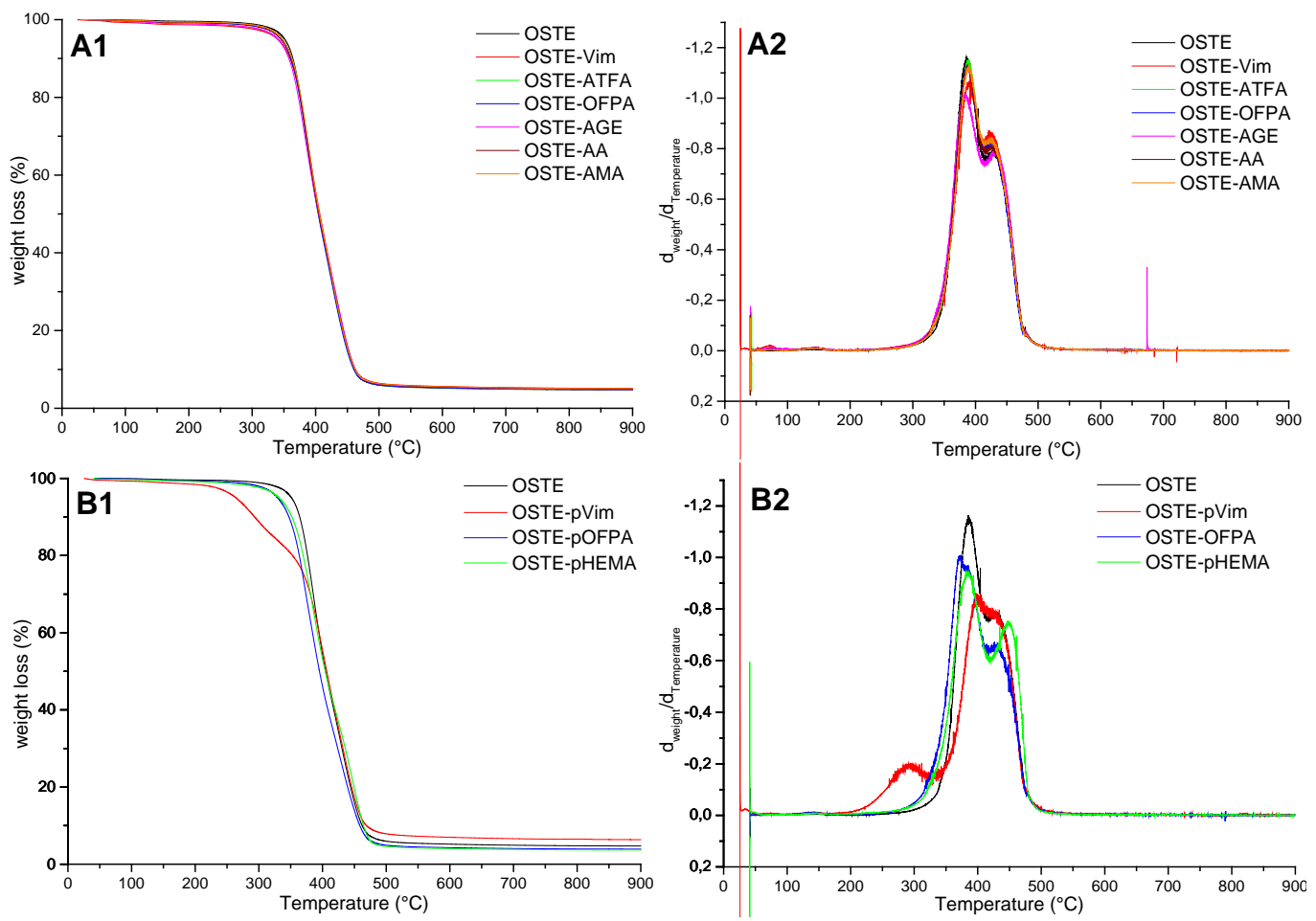

Figure S4 TGA Thermogram and its first derivative of pristine OSTE and functionalized particles via TEC (A1 and A2) and SCT-FRP (B1 and B2) 

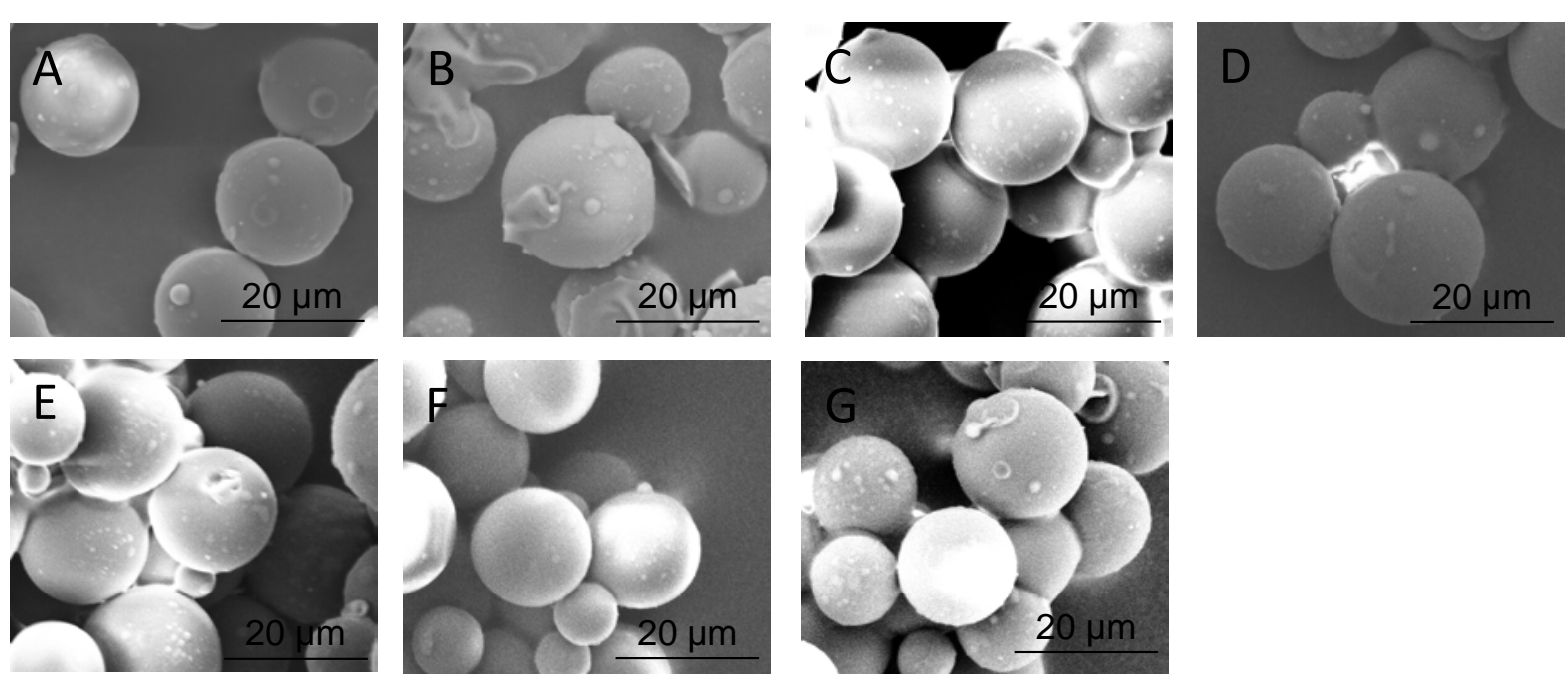

Figure S5 SEM images of OSTE particles containing different surface chemistry through TEC:

A) pristine OSTE, B) OSTE-Vim, C) OSTE-ATFA, D) OSTE-OFPA, E) OSTE-AGE, F) OSTE-AA, and G) OSTE-AMA
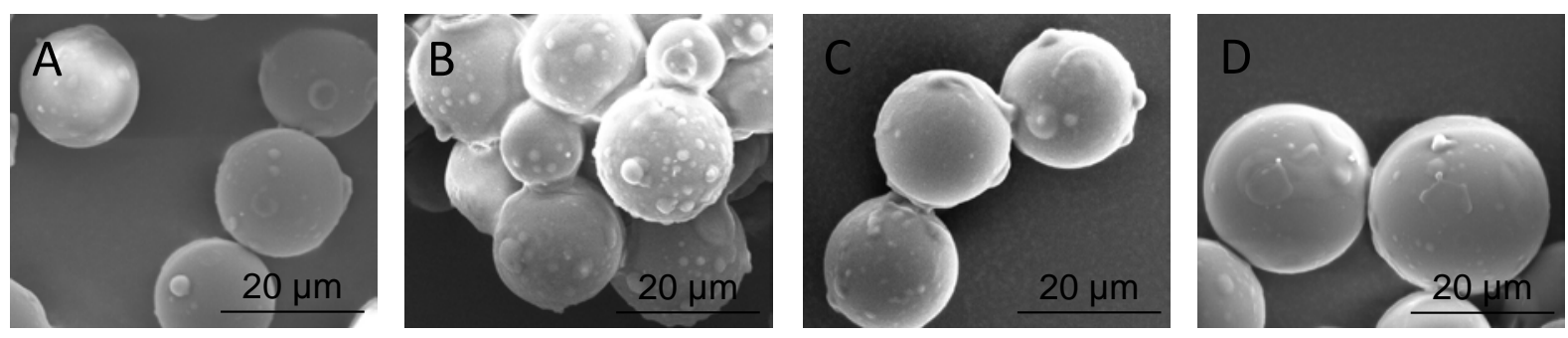

Figure S6 SEM images of OSTE particles containing different surface chemistry through SCT-

FRP: A) pristine OSTE, B) OSTE-pVim, C) OSTE-pOFPA, and D) OSTE-pHEMA 

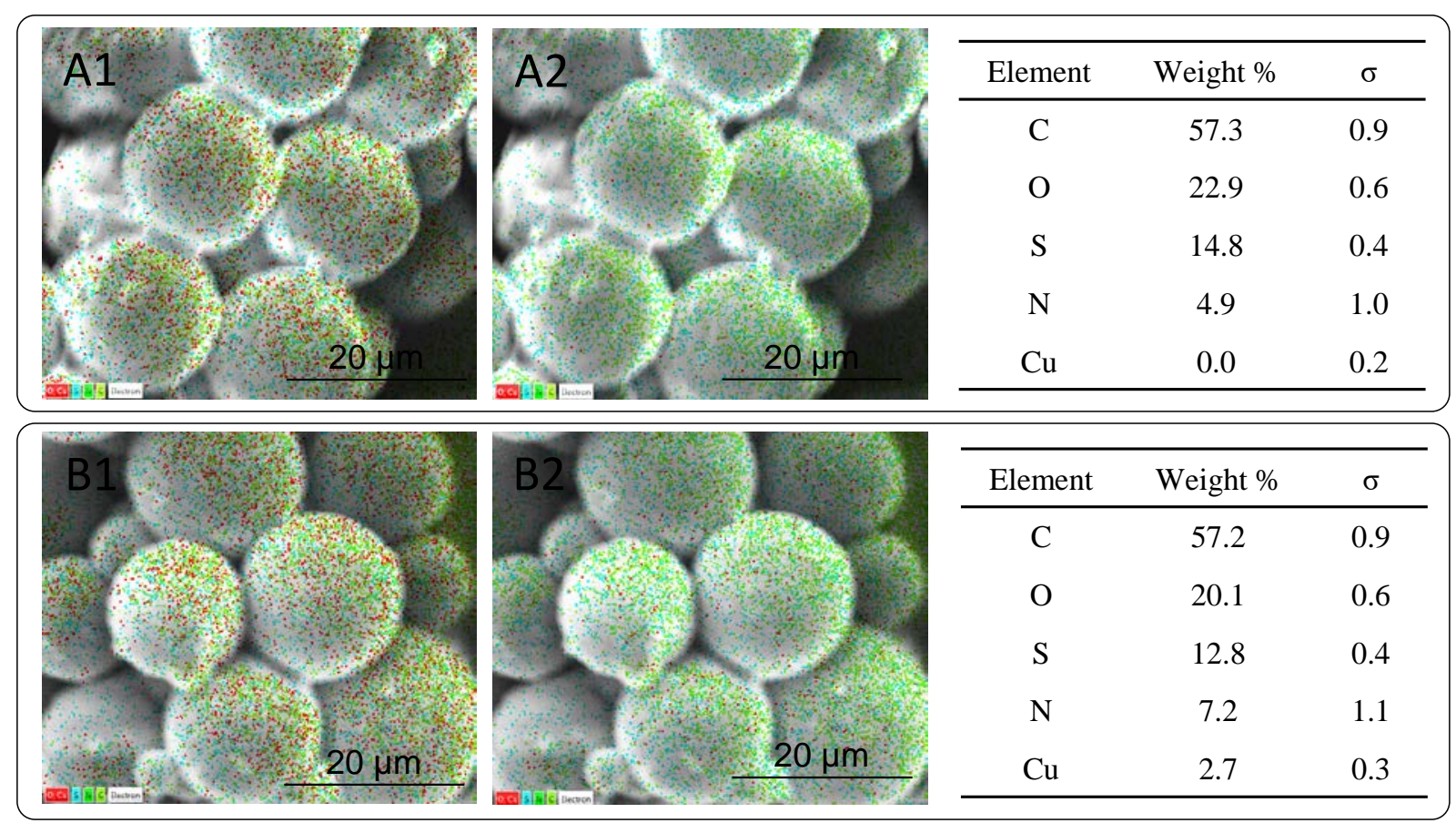

Figure S7 SEM images of OSTE-Vim-Cu (A1 and A2) and OSTE-pVim-Cu (B1 and B2)

particles, atom composition of particles was analyzed by using the EDX detector, which are depicted as colored dots within the images, including oxygen (A1 and B1) as well as excluding oxygen (A2 and B2), as well as in the tables 